STRUCTURAL BIOLOGY

ISSN 2059-7983

Received 16 May 2019

Accepted 12 July 2019

Edited by P. Langan, Oak Ridge National Laboratory, USA

₹ Current address: Department of Molecular Biology, Princeton University, Princeton, NJ 08544, USA.

Keywords: carbonic anhydrase; proton transfer; X-ray crystallography; carbonic anhydrase IX; neutron protein crystallography; perdeuteration; proton transfer.

PDB references: human carbonic anhydrase IX, protiated, 6rqn; 6rqq; H/D-exchanged, 6rqu; deuterated, 6rqw

Supporting information: this article has supporting information at journals.iucr.org/d

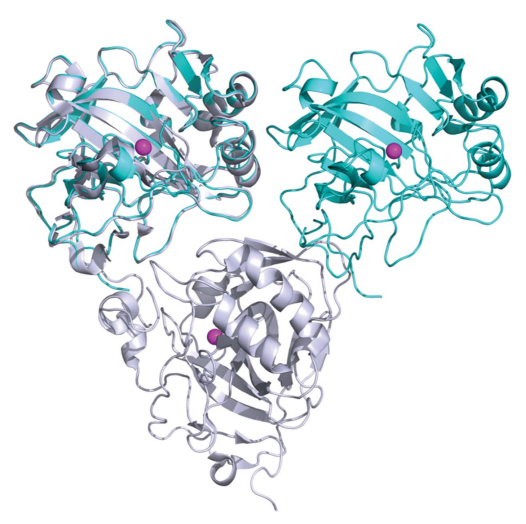

OPEN $\odot$ ACCESS

\section{Structural comparison of protiated, H/D-exchanged and deuterated human carbonic anhydrase IX}

\author{
K. Koruza, ${ }^{a}$ B. Lafumat, ${ }^{a}$ M. Nyblom, ${ }^{a}$ B. P. Mahon, ${ }^{b} \ddagger$ W. Knecht, ${ }^{a}$ R. McKenna ${ }^{b}$ \\ and S. Z. Fisher ${ }^{\mathrm{a}, \mathrm{c} *}$ \\ ${ }^{\mathrm{a}}$ Department of Biology and Lund Protein Production Platform, Lund University, Sölvegatan 35, 22362 Lund, Sweden, \\ ${ }^{\mathbf{b}}$ Department of Biochemistry and Molecular Biology, University of Florida, Gainesville, FL 32610, USA, and ' ${ }^{\text {SScientific }}$ \\ Activities Division, European Spallation Source ERIC, Odarslövsvägen 113, 22484 Lund, Sweden. *Correspondence \\ e-mail: zoe.fisher@esss.se
}

Human carbonic anhydrase IX (CA IX) expression is upregulated in hypoxic solid tumours, promoting cell survival and metastasis. This observation has made CA IX a target for the development of CA isoform-selective inhibitors. To enable structural studies of CA IX-inhibitor complexes using X-ray and neutron crystallography, a CA IX surface variant $\left(\mathrm{CA} \mathrm{IX}_{\mathrm{Sv}}\right.$; the catalytic domain with six surface amino-acid substitutions) has been developed that can be routinely crystallized. Here, the preparation of protiated (H/H), H/D-exchanged (H/D) and deuterated (D/D) CA IX $\mathrm{SV}_{\mathrm{SV}}$ for crystallographic studies and their structural comparison are described. Four CA IX $\mathrm{X}_{\mathrm{SV}} \mathrm{X}$-ray crystal structures are compared: two $\mathrm{H} / \mathrm{H}$ crystal forms, an $\mathrm{H} / \mathrm{D}$ crystal form and a D/D crystal form. The overall active-site organization in each version is essentially the same, with only minor positional changes in active-site solvent, which may be owing to deuteration and/or resolution differences. Analysis of the crystal contacts and packing reveals different arrangements of $\mathrm{CA}_{\mathrm{I}} \mathrm{X}_{\mathrm{Sv}}$ compared with previous reports. To our knowledge, this is the first report comparing three different deuteriumlabelled crystal structures of the same protein, marking an important step in validating the active-site structure of $\mathrm{CA} \mathrm{IX}_{\mathrm{SV}}$ for neutron protein crystallography.

\section{Background}

Carbonic anhydrases (CAs) are zinc-containing metalloenzymes that catalyze the reversible hydration of $\mathrm{CO}_{2}$ to form $\mathrm{HCO}_{3}^{-}$and $\mathrm{H}^{+}$. The first step of the reaction in the hydration direction results in a water molecule bound to the zinc that has to be deprotonated to generate a zinc-bound $\mathrm{OH}^{-}$for subsequent reactions. The excess $\mathrm{H}^{+}$is transported via an ordered hydrogen-bonded water network to a protonshuttling residue, His64 (CA II numbering), that ultimately delivers the $\mathrm{H}^{+}$to the bulk solvent (Coleman, 1967; Silverman \& McKenna, 2007). Numerous X-ray and neutron crystallographic studies have shed light on the catalytic mechanism of $\mathrm{CA}$ and have provided insights into the details of the finely tuned active site that supports $\mathrm{CO}_{2}$ hydration and rate-limiting proton transfer at very high rates $\left(k_{\mathrm{cat}}=10^{6} \mathrm{~s}^{-1}\right.$; Kim et al., 2016; Fisher et al., 2007, 2011; Domsic \& McKenna, 2010).

There are 15 expressed CA isoforms in humans that show diversity in expression between tissues and organs, supporting a range of physiological functions. One of the isoforms, CA IX, has limited expression in healthy tissues but is upregulated in aggressive tumours, with its expression being controlled by hypoxia (Pastorek \& Pastorekova, 2015). CA IX is a multidomain membrane-bound protein, with its catalytic CA domain facing extracellularly (Langella et al., 2018; Alterio et 
al., 2009). CA IX upregulation is part of a number of cancercell adaptions to hypoxia and is thought to occur in response to the lowering of $\mathrm{pH}$ in the cancer extracellular environment. The tumour $\mathrm{pH}$ environment is adapted from a physiological pH of 7.4 to as low as 6.0 (Mahon et al., 2015; Pastorek \& Pastorekova, 2015). This acidification promotes metastasis, most likely through protease activation and degradation of the extracellular matrix. A meta-study of patient outcomes showed very poor patient prognosis when positive for CA IX expression (Kuijk et al., 2016). For these reasons CA IX is a promising target for cancer detection and therapy, but high sequence conservation among human CAs (30-80\% aminoacid identity) results in indiscriminate binding of the currently available regime of clinically used CA inhibitors. Hence, there is a recognized need to develop isoform-specific inhibitors that inhibit CA IX strongly while ideally not inhibiting the other CAs (Pinard et al., 2015; Mahon et al., 2015).

Recent work by Mahon and coworkers reported the biophysical characterization and first X-ray crystal structure of a surface-modified variant of CA IX (CA IX Sv; $_{\text {; }}$ Mahon et al., 2016). CA IX $\mathrm{SV}_{\mathrm{SV}}$ contains only the catalytic domain of CA IX, with the intracellular, transmembrane and PG domains removed. In addition to truncating the full-length protein, six surface mutations (C174S, L180S, M360S, A210K, A258K and F259Y) were also introduced. These were chosen to remove a disulfide bond, to reduce surface hydrophobicity and to promote crystallization based on the crystal contacts in CA II (Mahon et al., 2016). The native, full-length protein is produced in insect cells in low yields, and is not stable or very soluble (Alterio et al., 2009). In this manuscript, and in the models deposited in the PDB, we use CA IX numbering. All residues mentioned in the text, tables and figures are for $\mathrm{CA}$ IX.

Endogenous CA IX is functional in a lower $\mathrm{pH}$ environment compared with other CAs. As such, Mahon and coworkers measured the thermal stability and catalytic parameters of CA IX under different $\mathrm{pH}$ conditions and demonstrated its adaptation to low $\mathrm{pH}$, giving rise to its structural and functional stability at $\mathrm{pH}$ values as low as 5.0. The $\mathrm{p} K_{\mathrm{a}}$ of the $\mathrm{H}^{+}$donor and acceptor groups in the active site are also decreased compared with CA II, indicating an ability to retain enzymatic activity at a $\mathrm{pH}$ of $\sim 6$ (Mahon et al., 2016).

Owing to the need for the development of CA isoformspecific inhibitors against CA IX, and to gain a deeper understanding of its active-site architecture, our goal is to utilize joint neutron and X-ray structures of CA IX $\mathrm{SV}_{\mathrm{SV}}$ alone and in inhibitor complexes to fine-tune compounds to preferentially bind CA IX over CA II (Langan \& Chen, 2013; Aggarwal et al., 2013; Kovalevsky et al., 2018). Previous neutron crystallographic studies of CA II in complex with clinically used inhibitors (for example brinzolamide, ethoxzolamide and acetazolamide) revealed the role and importance of water and hydrogen bonds in mediating ligandbinding interactions (Kovalevsky et al., 2018; Fisher et al., 2012). In preparation for future neutron protein crystallographic studies of experimental inhibitors binding to CA IX, we expressed unlabelled $\mathrm{CA}$ IX $\left(\begin{array}{lll}\mathrm{H} / \mathrm{H} & \mathrm{CA} & \left.\mathrm{IX}_{\mathrm{SV}}\right)\end{array}\right)$ and performed H/D exchange (H/D CA IX $\left.\mathrm{SV}_{\mathrm{SV}}\right)$ on preformed crystals. We also expressed deuterated protein (D/D CA IX $\left.\mathrm{SV}_{\mathrm{SV}}\right)$ for crystallization (Fisher et al., 2014; Koruza, Lafumat, Végvári et al., 2018; Blakeley et al., 2015).

There are several studies that have compared the properties, activities and X-ray crystal structures of unlabelled $(\mathrm{H} / \mathrm{H})$ and perdeuterated (D/D) versions of the same protein: cholesterol oxidase, haloalkane dehalogenase and arginase I (Golden et al., 2015; Liu et al., 2007; Di Costanzo et al., 2007). These studies all showed minimal structural effects owing to perdeuteration (D/D). However, we could not find studies in which X-ray crystal structures of $H / D$-exchanged versions were also included in the analysis. As H/D exchange is the most commonly used form of deuterium labelling for neutron protein crystallographic studies, it is important to determine and verify whether labelling by itself has any effect on the crystal structure. Here, we present a comparative structural analysis of three different isotopically labelled $(\mathrm{H} / \mathrm{H}, \mathrm{H} / \mathrm{D}$ and $\mathrm{D} / \mathrm{D})$ forms of CA IX $\mathrm{SV}$ and show that the overall fold and the active-site side-chain conformations are mostly unaffected. However, there are some subtle changes in solvent positioning that may be owing to deuteration effects and/or to differences in the resolutions of the structure determinations. We also analyzed the crystallographic monomer-to-monomer contacts and packing for different space groups of $\mathrm{CA} \mathrm{IX}_{\mathrm{SV}}$ compared with previous reports.

\section{Materials and methods}

In this study, we use three designations to indicate protiated, $\mathrm{H} / \mathrm{D}$-exchanged and deuterated status: $\mathrm{H} / \mathrm{H}$ means protiated protein in protiated buffer, H/D refers to protiated protein that was subjected to vapour $H / D$ exchange after crystallization and $\mathrm{D} / \mathrm{D}$ is deuterated protein that was purified in protiated buffers and then subjected to back-exchange in solution to recover any lost D atoms. For all studies we used a construct created by Mahon et al. (2016) containing the catalytic domain of CA IX with six surface mutations introduced (C174S, L180S, M360S, A210K, A258K and F259Y) that was engineered to facilitate crystallization $\left(\mathrm{CA} \mathrm{IX}_{\mathrm{SV}}\right)$.

\subsection{Expression and purification of protiated $C A I X_{S V}$}

$\mathrm{CA}_{\mathrm{IX}}$ Sv production has been described in detail elsewhere (Koruza, Lafumat, Végvári et al., 2018; Mahon et al., 2016). Briefly, CA IX Sv $_{\text {was expressed in Escherichia coli BL21 }}$ (DE3) cells under kanamycin selection (final concentration of $50 \mu \mathrm{g} \mathrm{ml}^{-1}$ ) in a shaking incubator at $37^{\circ} \mathrm{C}$. The cells were grown to an $\mathrm{OD}_{600}$ of $\sim 1.0$ and expression was induced by the addition of $1 \mathrm{~m} M$ isopropyl $\beta$-D-1-thiogalactopyranoside (IPTG) in the presence of $1 \mathrm{mM} \mathrm{ZnSO}_{4}$. After $4 \mathrm{~h}$ the cells were harvested by centrifugation $(5000 \mathrm{~g}$ for $20 \mathrm{~min}$ ) and the cell pellets were frozen at $-20^{\circ} \mathrm{C}$. The cell pellets were lysed by thawing at room temperature in $0.2 \mathrm{M}$ sodium sulfate, Tris$\mathrm{HCl} \mathrm{pH} 9$ and then stirring in the cold room for $\sim 3 \mathrm{~h}$ in the presence of $20 \mathrm{mg}$ lysozyme and $1 \mathrm{mg}$ DNaseI. Clarified lysates were prepared by centrifugation at $50000 \mathrm{~g}$ for $60 \mathrm{~min}$ 
at $4{ }^{\circ} \mathrm{C}$. Affinity chromatography using $p$-aminomethylbenzenesulfonamide resin (Sigma-Aldrich) (wash buffer 1, $0.2 \mathrm{M}$ sodium sulfate, Tris- $\mathrm{HCl} \mathrm{pH}$ 9; wash buffer 2, 0.2 $\mathrm{M}$ sodium sulfate, Tris- $\mathrm{HCl} \mathrm{pH} \mathrm{7;} \mathrm{elution} \mathrm{buffer,} 0.4 M$ sodium azide, $50 \mathrm{~m} M$ Tris- $\mathrm{HCl} \mathrm{pH}$ 7.8) was followed by size-exclusion chromatography (50 $\mathrm{m} M$ Tris- $\mathrm{HCl} \mathrm{pH} \mathrm{7.8,100} \mathrm{mM} \mathrm{NaCl).} \mathrm{CA}$ $\mathrm{IX}_{\mathrm{SV}}$ elutes from the size-exclusion column in two peaks corresponding to dimeric and monomeric forms (Koruza, Lafumat, Végvári et al., 2018).

Peak fractions corresponding to monomeric $\mathrm{CA} \mathrm{IX}_{\mathrm{sv}}$ were pooled and concentrated using Amicon Ultra Centrifugal Filter Units (Merck) with a molecular-weight cutoff of $10 \mathrm{kDa}$ and were analyzed by sodium dodecyl sulfate-polyacrylamide gel electrophoresis (SDS-PAGE) to estimate their purity. The protein was concentrated to a final concentration of $17 \mathrm{mg} \mathrm{ml}^{-1}$ for crystallization.

\subsection{Expression and purification of deuterated $C A I X_{S V}$}

Deuterated CA IX $\mathrm{SV}_{\mathrm{SV}}$ was expressed in E. coli BL21 (DE3) cells according to a protocol described elsewhere (Koruza, Lafumat, Végvári et al., 2018). Briefly, cells were pre-grown in LB Broth (Miller) (Difco) at $37^{\circ} \mathrm{C}$. The growth medium was then exchanged in the middle of the exponential phase for the same volume of deuterated ModC1 medium supplemented with $2 \%$ unlabelled glycerol (Koruza, Lafumat, Végvári et al., 2018; Duff et al., 2015). Upon dilution in the deuterated medium, the cells were allowed to recover for $1 \mathrm{~h}$ at $37^{\circ} \mathrm{C}$ while shaking at $120 \mathrm{rev} \min ^{-1}$. Following the adaptation period, the temperature was decreased to $25^{\circ} \mathrm{C}$ and shaking was increased to $200 \mathrm{rev} \mathrm{min}^{-1}$. Protein expression was induced by the addition of IPTG in the presence of $1 \mathrm{~m} M$ zinc sulfate. The cells were harvested after $18 \mathrm{~h}$ by centrifugation and stored at $-20^{\circ} \mathrm{C}$. Deuterated CA IX $\mathrm{IS}_{\mathrm{Sv}}$ was purified as described for the protiated form in Section 2.1.

\subsection{CA IX $X_{S V}$ crystallization optimization and H/D exchange}

Crystallization drops were prepared using both the hangingdrop and sitting-drop vapour-diffusion methods after a lengthy optimization procedure as described elsewhere (Koruza, Lafumat, Nyblom et al., 2018). Briefly, crystals were initially grown for the preparation of seed stocks using a 1:1 ratio of protein solution $\left(17 \mathrm{mg} \mathrm{ml}^{-1} \mathrm{H} / \mathrm{H} \mathrm{CA} \mathrm{IX}\right.$ Sv $)$ and $30 \%(w / v)$ PEG 4000, $0.1 M$ Tris- $\mathrm{HCl}$ pH 8.5, $0.2 M$ sodium acetate or $0.2 M$ ammonium formate. These crystals were then sacrificed for seed-stock preparation in the mother liquor as described in the instructions for the Seed Bead Kit (Hampton Research; https://www.hamptonresearch.com). Crystallization was repeated using a 3:2:1 ratio of protein:precipitant:seed stock in drop volumes of between 6 and $24 \mu$ l. With seeding, crystals appeared within a week. Both protiated and deuterated $\mathrm{CA} \mathrm{IX}_{\mathrm{SV}}$ were crystallized using protiated buffers. Crystals of $\mathrm{H} / \mathrm{H} \mathrm{CA} \mathrm{IX}_{\mathrm{Sv}}$ were used without further manipulation. To prepare H/D CA IX $\mathrm{S}_{\mathrm{SV}}$ and D/D CA IX $\mathrm{X}_{\mathrm{SV}}$ crystals, the reservoir solution was removed and replaced with a deuterated version. The drops were then resealed and allowed to $\mathrm{H} / \mathrm{D}$-exchange for several weeks prior to X-ray data collection. Prior to cooling the crystals by plunging them into liquid nitrogen, they were cryoprotected by dipping them into reservoir solution supplemented with $20 \%$ glycerol. For the H/D and D/D crystals, deuterated glycerol was used for cryoprotectant preparation.

\subsection{Crystallographic data collection and structure refinement}

Two diffraction data sets for $\mathrm{H} / \mathrm{H}$ CA IX $\mathrm{XV}_{\text {Sv }}$ were collected at $100 \mathrm{~K}$ on the FIP-BM30A beamline (Roth et al., 2002) at the European Synchrotron Radiation Facility (ESRF), Grenoble, France and on the BioMAX beamline at MAX IV Laboratory, Lund, Sweden. The H/D and D/D CA IX collected on the BioMAX beamline at MAX IV Laboratory, Lund, Sweden.

Data processing was performed using the autoPROC software package (Vonrhein et al., 2011). The automated workflow script mainly uses $X D S$ (Kabsch, 2010) as the data-processing and scaling software and POINTLESS for space-group determination. For two of the data sets (H/D and D/D) 3600 images were collected. The images were processed in batches to find a cutoff where radiation damage impacts the data quality. However, data processing and subsequent model refinement showed that it was best to use the full data sets.

The phases for all of the X-ray data were obtained by molecular replacement in Phaser (McCoy et al., 2007) using PDB entry 5dvx (Mahon et al., 2016) as a search model. The models were initially rigid-body refined in Phaser, followed by restrained refinement in the PHENIX suite (Adams et al., 2011). For all data sets, a bulk-solvent correction and a free $R$-factor monitor (calculated with $5 \%$ of randomly chosen reflections) were applied throughout the refinement. $2 F_{\mathrm{o}}-F_{\mathrm{c}}$ and $F_{\mathrm{o}}-F_{\mathrm{c}}$ map interpretation and manual model building was performed using Coot (Emsley et al., 2010). For the apparently larger $P 2_{1}$ unit cell, both chains were refined without applying noncrystallographic symmetry (NCS).

Figures were generated using PyMOL (Schrödinger; http:// www.pymol.org). The CA IX $\mathrm{SV}$ structures were deposited in the RCSB Protein Data Bank with the following accession codes: 6rqn, 6rqq, 6rqu and 6rqw. Data-collection and refinement statistics are summarized in Table 1. Dimer-interface analysis, buried surface-area calculation and mapping of interactions were performed in $P y M O L$ and Coot and using the PDBePISA server (Emsley et al., 2010; Krissinel \& Henrick, 2007).

\section{Results and discussion}

\subsection{Crystallography}

Crystals for X-ray data collection were obtained in both hanging-drop and sitting-drop vapour-diffusion setups. Microseeding into drop volumes varying between 3 and $10 \mu \mathrm{l}$ produced crystals within 1-2 weeks. There were noticeable and reproducible differences in the number, size and quality of the crystals depending on the deuteration status of the protein (Fig. 1; Koruza, Lafumat, Végvári et al., 2018). For the crystals 
used in this study the volumes ranged from 0.01 to $0.03 \mathrm{~mm}^{3}$. The largest CA IX $\mathrm{SV}_{\mathrm{SV}}$ crystal that we obtained was $0.8 \mathrm{~mm}^{3}$ and efforts to scale up and increase the volume continue (see Fig. 5 in Koruza, Lafumat, Nyblom et al., 2018). We obtained crystals in space group $P 2_{1}$ with two apparently different unit cells labelled 'small' (unit-cell parameters $a=44.5, b=65.4$, $c=46.7 \AA, \beta=115.1^{\circ}$ ) and 'big' (unit-cell parameters $a=48.9$, $\left.b=65.1, c=76.3 \AA, \beta=92.86^{\circ}\right)$. Data from $\mathrm{H} / \mathrm{H}$ crystals were initially collected on the FIP-BM30 beamline at the ESRF and they were shown to belong to a different space group to the previously reported $P 2_{1} 2_{1} 2_{1}$ (Mahon et al., 2016). Subsequent data collection from $\mathrm{H} / \mathrm{H}, \mathrm{H} / \mathrm{D}$ and $\mathrm{D} / \mathrm{D}$ crystals on the
BioMAX beamline at MAX IV Laboratory revealed that $\mathrm{H} / \mathrm{H}$ also indexed as space group $P 2_{1}$ but with a 'big' unit cell (Table 1). The other $H / D$ and $D / D$ crystals were both in the 'small' $P 2_{1}$ unit cell, the same as the first one we determined from ESRF data. A summary of data-set and refinement statistics is shown in Table 1 . The crystals all diffracted with good statistics and the structures were determined to $1.77-1.28 \AA$ resolution.

\subsection{Space-group and crystal-packing analysis}

The small $P 2_{1}$ monoclinic unit cell contained two CA IX $\mathrm{SV}$ chains (one per asymmetric unit) with a volume of $123080 \AA^{3}$.

Table 1

Data-collection and model-refinement statistics for CA IX $\mathrm{Sv}_{\mathrm{Sv}}$

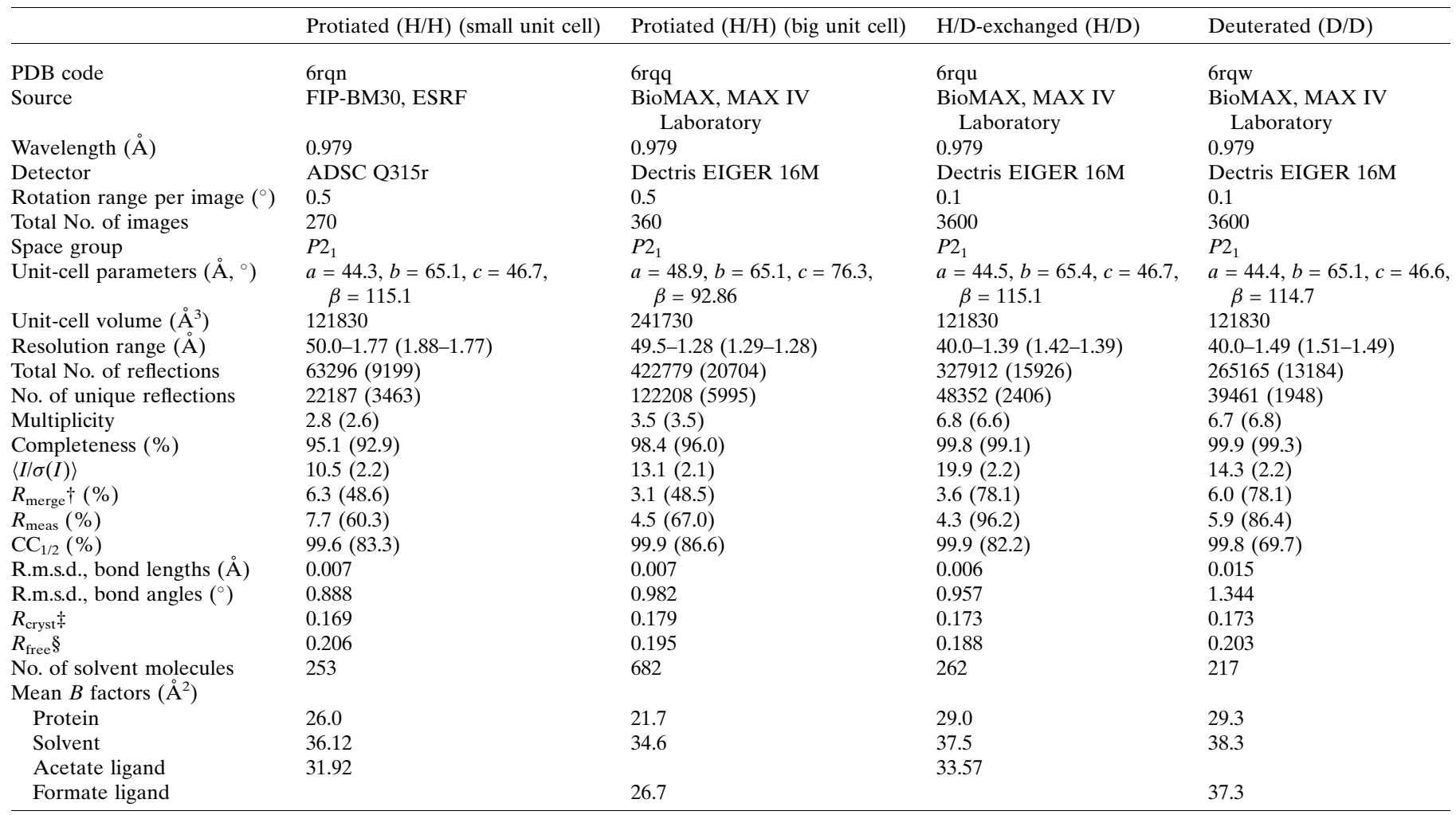

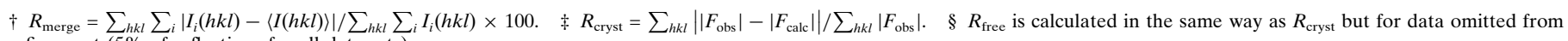
refinement (5\% of reflections for all data sets)

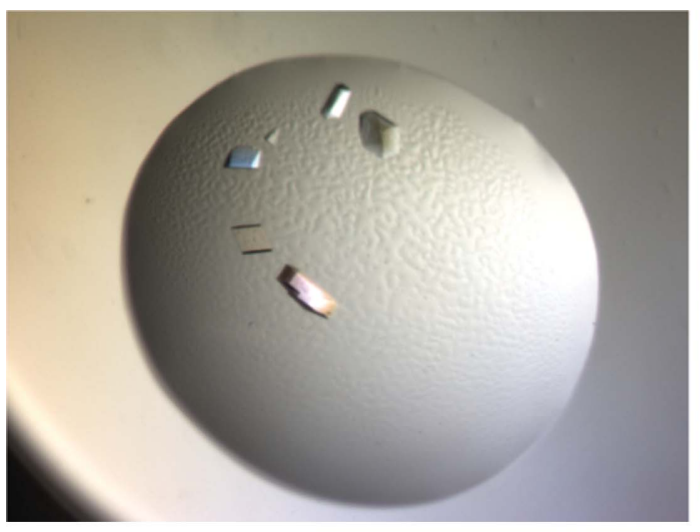

(a)

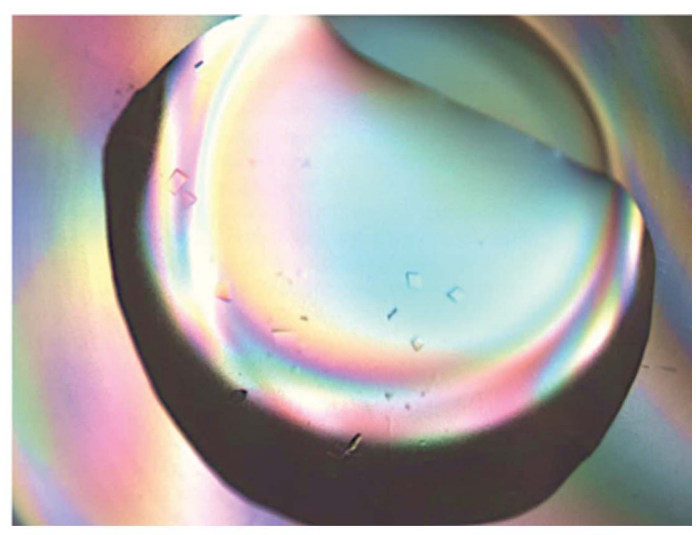

(b)

Figure 1

Photographs of hanging-drop and sitting-drop vapour-diffusion setups for producing $(a)$ protiated and $(b)$ deuterated CA IX $\mathrm{Sv}_{\mathrm{Sv}}$. Both of the drops shown here are $10 \mu \mathrm{l}$ in volume. 
The other $\mathrm{H} / \mathrm{H}$ crystal unit cell processed as a 'big' $P 2_{1}$ cell, but is in fact a doubled 'small' unit cell with the two chains in the apparent ASU related by translational NCS of $\frac{1}{2}, 0, \frac{1}{2}$. So, while theoretically redundant to the 'small' $\mathrm{H} / \mathrm{H}$ structure in terms of packing and contacts, the crystallization conditions were slightly different and we do see a different ligand bound in the active site (discussed later in Section 3.3). For these reasons we show the data statistics in Table 1 and discuss the active site in Section 3.3 and Fig. 5. The CA IX $\mathrm{Sv}_{\mathrm{Sv}}$ packing arrangements are shown in Fig. 2, with symmetry-related molecules shown in grey.

Previous biophysical studies of CA IX have established it to be dimeric both in vivo and in vitro, although the precise organization of the native dimer is unknown (Hilvo et al., 2008; Li et al., 2011). A dimer of dimers was observed in the first published crystal structure of the CA IX catalytic domain; it was produced using a baculovirus expression-vector system and the structure was determined in space group $P 6_{1}$ (PDB entry 3iai; unit-cell parameters $a=b=144.2, c=208.9 \AA$; Alterio et al., 2009). In this structure protein dimerization was mediated by an intermolecular disulfide bond involving Cys174 (position 41 in CA II) and was proposed to be a physiologically relevant quaternary structure. Interestingly, in the same report a Cys174Ser CA IX variant crystallized with the same packing and dimeric interface but without the disulfide bond (Alterio et al., 2009). Recently, another study reported a CA IX structure, this time determined from protein expressed in yeast, that crystallized in space group $H 3$ (PDB entry 6fe0; unit-cell parameters $a=b=152.9, c=171.5 \AA$; Kazokaitè et al., 2018). In addition to these studies, a crystal structure of CA IX $\mathrm{SV}_{\mathrm{SV}}$ was also determined in space group $P 22_{1} 2_{1}$ (PDB entry 5dvx; unit-cell parameters $a=57.9$, $b=102.7, c=108.9 \AA$ ). This structure also had two NCS chains in the ASU, however, the arrangement did not correspond to the previous reports of the native CA IX. This was most likely owing to the Cys174 residue being mutated to a serine, but the unit cell and crystal packing were also different (Mahon et al., 2016). As such, this smaller orthorhombic space-group unit cell was more suitable for neutron studies than the previously published hexagonal cell, and we pursued the orthorhombic form for our studies. Consideration of the unit-cell parameters in neutron protein crystallography experiments (normally up to a maximum of $150 \AA$ ) is related to the limitations of the current flux of neutron sources as well as the layout of macromolecular beamlines to resolve larger unit-cell parameters (Meilleur et al., 2018; O'Dell et al., 2016). To be able to obtain reasonable diffraction data (better than $2 \AA$ resolution) from a crystal with a large unit cell, it is necessary to also optimize the overall crystal volume (Tanaka, 2019). Despite extensive efforts to reproduce these crystals of CA IX $\mathrm{X}_{\mathrm{SV}}$, we instead obtained two new and seemingly different monoclinic $P 2_{1}$ crystals (Fig. 1, Table 1). The 'small' monoclinic $P 2_{1}$ crystal form is isomorphous to the deuterium-labelled $\mathrm{CA} \mathrm{IX}_{\mathrm{SV}}$ crystal form. In addition, there is only one $\mathrm{CA}_{\mathrm{IX}} \mathrm{Sv}$ per
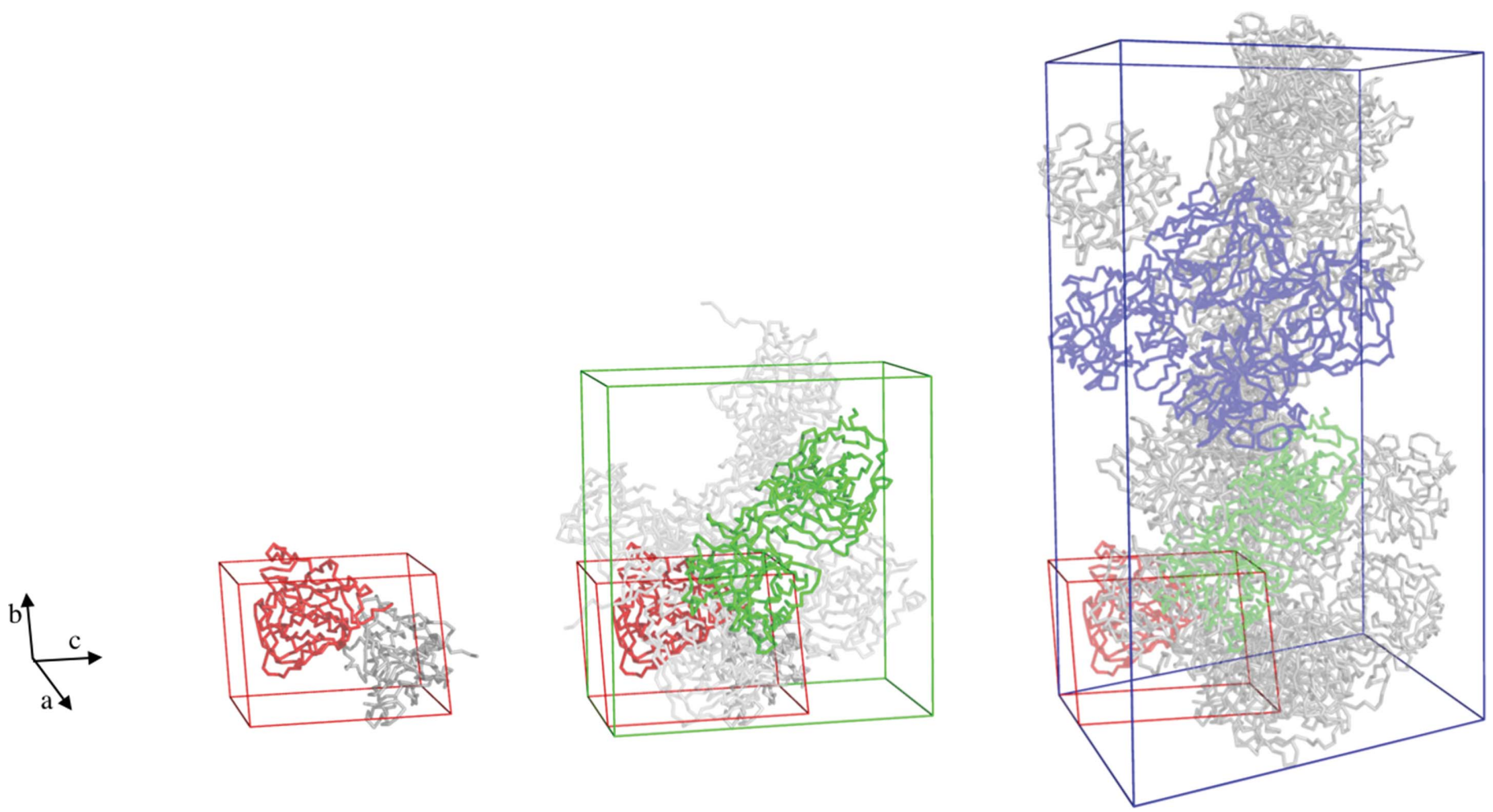

Figure 2

Crystal packing diagrams of CA IX $\mathrm{SV}$ in $P 2_{1}$ (this work) and $P 2_{1} 2_{1} 2_{1}$ (PDB entry 5dvx), and CA IX in $P 6_{1}$ (PDB entry 3 iai) unit cells. The monomer in the ASU from the small $P 2_{1}$ is shown as a red ribbon in all diagrams for reference. The two chains in the ASU unit of $P 2_{1} 2_{1} 2_{1}$ dimer are in green and in blue for $P 6_{1}$. 
Table 2

Crystallographic interchain interactions in CA IX $\mathrm{Sv}_{\mathrm{Sv}}$ : comparison between $P 2_{1}$ (this work) and $P 2_{1} 2_{1} 2_{1}$ (PDB entry $5 \mathrm{dvx}$ ).

Hydrogen-bond and salt-bridge distances are shown in parentheses and indicate donor-acceptor (heavy atom) distances. $A$ chains are listed first. Interactions longer than $3.4 \AA$ were excluded. Analysis was performed using the PBDePISA server and was verified visually in Coot (Krissinel \& Henrick, 2007; Emsley et al., 2010). Mutated residues in CA IX $\mathrm{SV}$ are shown in bold.

\begin{tabular}{|c|c|c|}
\hline $\begin{array}{l}P 2_{1} \text { 'small' (this work): } \\
\text { monomer in asymmetric unit }\end{array}$ & $\begin{array}{l}P 2_{1} \text { 'big' (this work): } \\
\text { dimer in asymmetric unit }\end{array}$ & $\begin{array}{l}\left.P 2_{1} 2_{1} 2_{1} \text { (PDB entry } 5 \mathrm{dvx}\right) \text { : } \\
\text { dimer in asymmetric unit }\end{array}$ \\
\hline $\begin{array}{l}\text { Glu280-Glu192 via water }(2.6 \text { and } 2.8 \AA) \\
\text { Gln307-Arg323 }(2.9 \AA) \\
\text { Ser319-Glu305 }(2.7 \AA) \\
\text { Asp320-Gln307 }(2.9 \AA) \\
\text { Arg323-Glu298 }(3.2 \AA) \\
\text { Arg323-Thr306 }(3.2 \AA) \\
\text { Asn346-Glu192 }(3.1 \AA) \\
\text { Asn346-Gln307 }(2.8 \AA) \\
\text { Gln347-Glu305 }(2.8 \AA)\end{array}$ & $\begin{array}{l}\text { Glu297-Glu219 via water }(2.8 \text { and } 2.7 \AA) \\
\text { Glu297-Thr257 via water }(2.8 \text { and } 2.7 \AA) \\
\text { Glu301-Lys258 (-CO) }(3.4 \AA) \\
\text { His357- Pro175 via water }(3.0 \text { and } 3.4 \AA) \\
\text { Asp361-Pro216 (-CO) }(3.3 \AA) \\
\text { Asp368-Arg268 via water }(2.5 \text { and } 3.1 \AA) \\
\text { Asp368-Lys258 }(2.7 \AA) \\
\text { Asp368-Tyr259 via water }(3.2 \text { and } 3.0 \AA)\end{array}$ & $\begin{array}{l}\text { Arg167-Asp146 (-CO) }(3.1 \AA) \\
\text { Gln169-Pro148 }(-\mathrm{CO}) \text { via water }(2.7 \text { and } 2.8 \AA) \\
\text { Gly233 (N)-Glu305 (-CO) }(2.8 \AA) \\
\text { Glu242-Trp141 }(\mathrm{N})(3.2 \AA) \\
\text { Gly243 (-CO)-Gly367 (N) }(2.9 \AA) \\
\text { His244-Zn-His200 }(3.3 \AA) \\
\text { His244-Zn-Trp141 (N) }(3.3 \AA) \\
\text { His244-Glu302 }(2.8 \AA) \\
\text { Arg245 (N)-Glu302 }(2.9 \AA) \\
\text { Asp395 (-CO)-Val152 }(\mathrm{N})(2.9 \AA) \\
\text { Ser396-Ser153 via water }(2.6 \text { and } 2.6 \AA) \\
\text { Arg399-Asp263 (-CO) }(2.8 \AA) \\
\text { Arg399-Leu266 (-CO) }(2.7 \AA)\end{array}$ \\
\hline
\end{tabular}

asymmetric unit, which is useful when using neutrons for protein crystallography (Blakeley et al., 2015). For a complete list of crystal contacts, refer to Table 2.

Fig. 3 shows an overlay of the two chains in the monoclinic unit cell with the two chains in the orthorhombic ASU, with chain A from the latter as the reference. The monomer-tomonomer electrostatic contacts for both are listed in Table 2. There are more interactions mediating the interface in the orthorhombic chains, most of which are attributable to the $\mathrm{C}$ terminal residues wrapping around the dimer pair. The observation of multiple monomer-to-monomer arrangements suggests that CA IX has a strong propensity to dimerize, independent of intermolecular disulfide bonds, and it is not possible to infer which may be the relevant physiological dimer. The differences in arrangement and crystal packing between native CA IX and CA IX Sv $_{\text {are illustrated in Fig. } 2 .}$

The CA IX $\mathrm{SV}_{\mathrm{SV}}$ variant has six surface amino-acid substitutions compared with CA IX, which were chosen to optimize expression in E. coli and crystallization. The intention was to eliminate dimerization owing to disulfide-bond formation, to reduce surface hydrophobicity and to encourage possible crystal contacts based on CA II crystal contacts (Mahon et al., 2016). Hence, we wanted to investigate whether some of the amino-acid interactions involved in the crystal contacts in the $P 2_{1}$ or the $P 2{ }_{1} 2_{1} 2_{1}$ unit cell were affected by the six amino-acid substitutions. Figs. 4(a) and 4(b) shows the dimer in ribbon representation, with the substituted side chains depicted as sticks. From inspection of these structures, it was apparent that residues Lys258 and Tyr259 were involved in NCS dimerization in the big monoclinic $P 2_{1}$ unit cell reported here [Table 2, Fig. 4(b)]. In the small monoclinic $P 2_{1}$ and previously reported orthorhombic $P 2_{1} 2_{1} 2_{1}$ unit cells [Fig. $4(c)$ ] none of the aminoacid substitutions were involved in crystal contacts or NCS dimerization. It would therefore appear that the six surface residues that were changed do not drive dimerization but do have an important impact on the solubility and stability of the catalytic domain of CA IX.

\subsection{Active-site comparison of $\mathbf{H} / \mathbf{H}$ (small), $\mathbf{H} / \mathbf{H}$ (big), $\mathbf{H D}$ and DD CA IX $\mathrm{SV}_{\mathrm{SV}}$ structures}

When deuterating proteins for neutron studies, it is important to determine whether deuteration causes appreciable conformational effects in the resulting protein side chains and active-site solvent positioning. For the deuterated protein to be useful in structural studies it has to be representative of the physiological protiated protein: there should be no conformational changes. As noted before, the overall activesite arrangement of solvent and amino-acid residues appears

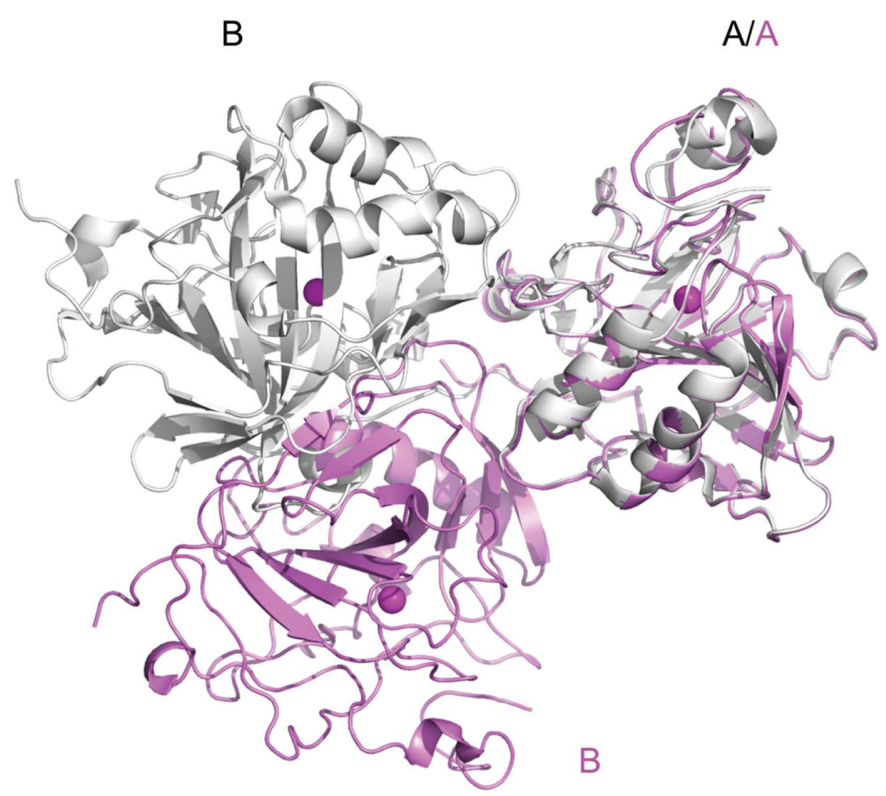

Figure 3

Overlay of the two chains in the unit cell of $P 2_{1}$ (this work) and the two chains in the ASU of $P 2_{1} 2_{1} 2_{1}$ (PDB entry 5dvx; Mahon et al., 2016) with chain $A$ as reference to illustrate differences in crystallographic organization. The chains for the small $P 2_{1}$ unit cell are shown as a pink cartoon while the two NCS chains in the ASU from $P 2_{1} 2_{1} 2_{1}$ are shown as a grey cartoon. Zinc atoms are shown as magenta spheres to indicate the location of the active site. 
to be largely unaffected, with an r.m.s.d. variation for all atoms when superimposing all four structures onto each other of less than $1 \AA$. We conducted a careful OMIT map analysis of the structures and found that in all four structures there are one of two ions bound to the zinc ion that come from the crystallization conditions: either acetate or formate (Fig. 5). Both formate and acetate are known inhibitory anions that bind to CAs and therefore their presence is not a surprise (Coleman, 1967; Håkansson et al., 1992; Alterio et al., 2009). Formate inhibits by displacing the so-called 'deep water' in the active site and binds in the same location as the carbon dioxide substrate (Fig. 5; Domsic \& McKenna, 2010), whereas acetate

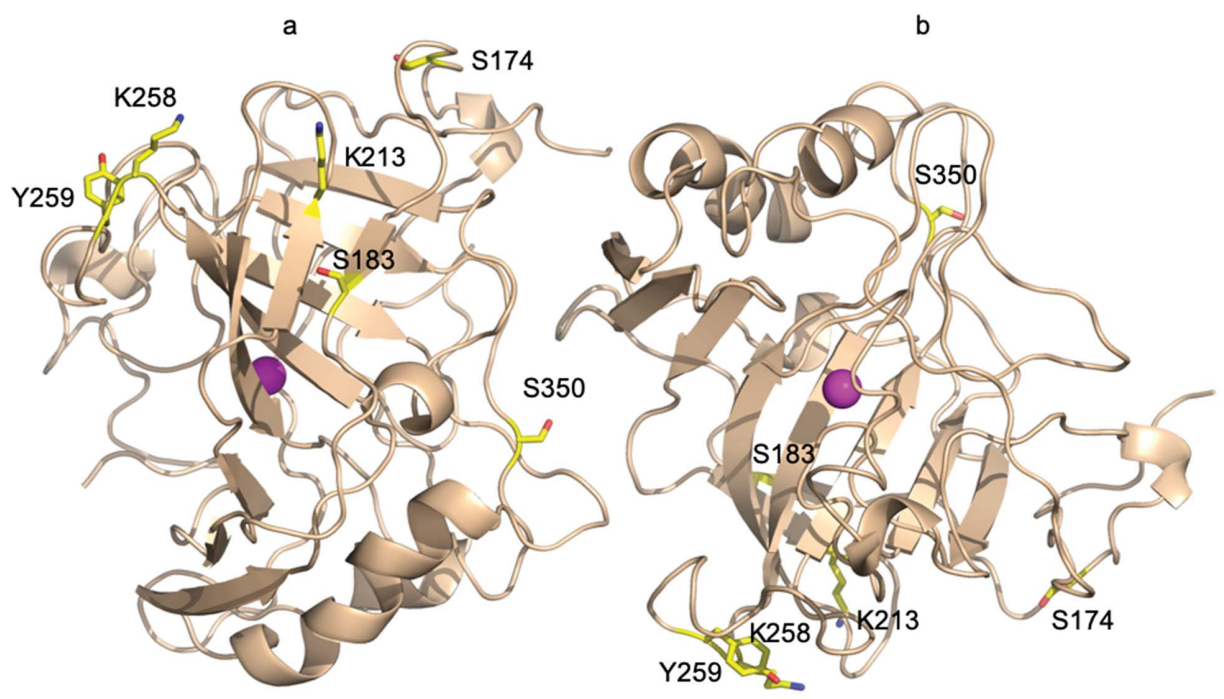

(a)

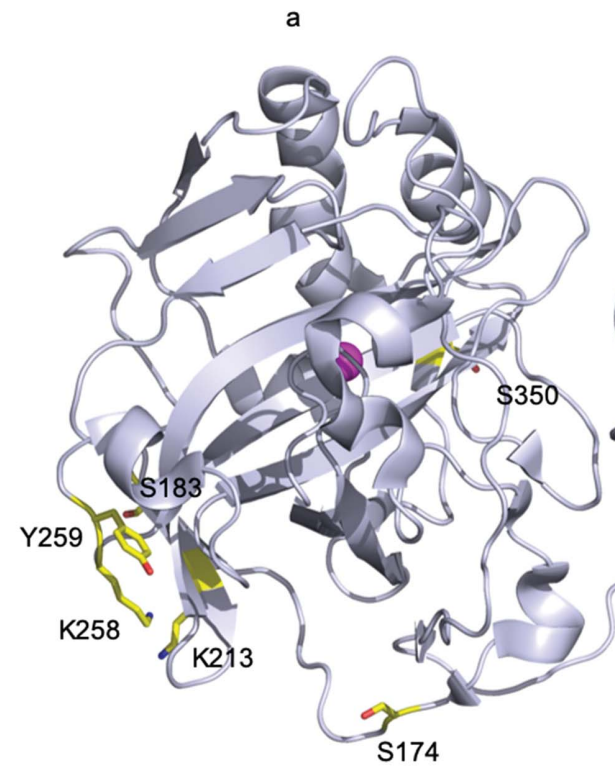

b

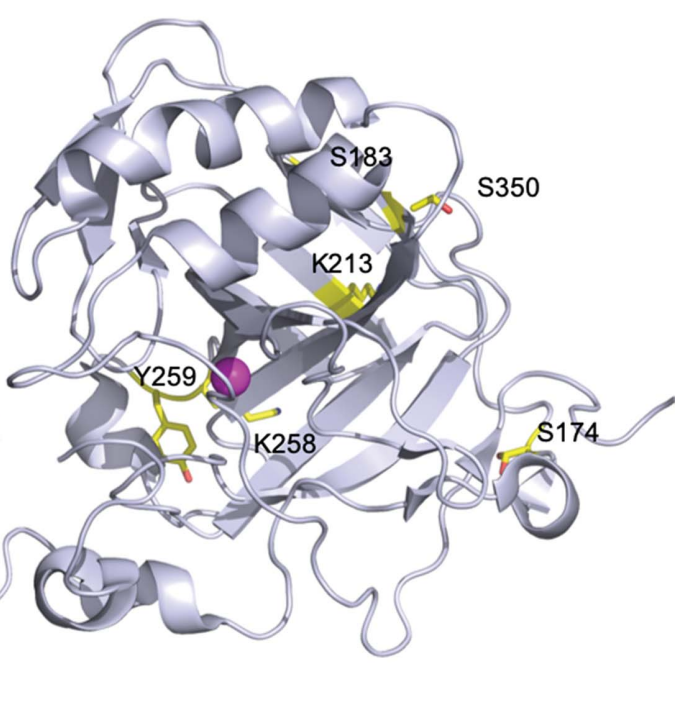

(b)

Figure 4

Comparison of crystallographic contacts in view of the locations of the six mutations present in CA IX $\mathrm{Sv}$. CA IX $\mathrm{SV}_{\mathrm{S}}$ is shown in cartoon representation, substituted amino acids (compare with CA IX) are depicted as yellow sticks, zinc is shown as a magenta sphere. (a) Crystallographic chains in the small $P 2_{1}$ unit cell (this work), (b) NCS chains in the ASU of the $P 2_{1} 2_{1} 2_{1}$ cell (PDB entry 5dvx; Mahon et al., 2016). replaces two waters, the deep water and the catalytic zincbound water, effectively presenting an inhibited active-site structure (Supplementary Fig. S1). We observed formate bound in the apparent 'big' cell in $\mathrm{H} / \mathrm{H}$ and the $\mathrm{D} / \mathrm{D}$ structures, while acetate was present in the small $\mathrm{H} / \mathrm{H}$ and the $\mathrm{H} / \mathrm{D}$ exchanged structures (Fig. 5). The presence of either ion does not seem to affect the overall active-site side-chain conformations, with the exception of small water rearrangements, which are reflected in the relative weak density for the activesite solvent molecule W2 [Fig. 5(a)]. In Figs. 5(b)-5(d) it can be seen that W2 has poor density at the same contouring and also has a higher refined crystallographic $B$ factor compared with the other active-site solvent molecules. The weak density of W2 is most apparent in the $\mathrm{D} / \mathrm{D}$ structure, where there is no density below the $1.5 \sigma$ level in the OMIT electron-density map. There are multiple possible explanations for this, including the presence of formate that may perturbate the water network or subtle effects caused as a result of protein deuteration.

Careful electron-density OMIT analysis of the proton-shuttling residue His200 shows it to be split between two conformations, termed the 'in' and the 'out' conformation in the CA II literature (Supplementary Fig. S2; Nair \& Christianson, 1991; Fisher et al., 2005). In the structure with PDB code 5dvx (CA $\left.\mathrm{IX}_{\mathrm{SV}}\right)$ the His 200 side chain was fully in the 'out' position and was $\pi$-stacked with Trp141 (Mahon et al., 2016). There is structural evidence that the preferred position of His64 in CA II is strongly affected by the $\mathrm{pH}$, with the 'out' conformation being dominant at low $\mathrm{pH}$ owing to charge repulsion between the charged His and the zinc (Fisher et al., 2005). However, all the monoclinic structures reported here and the previously reported orthorhombic forms were all determined from crystals grown at $\mathrm{pH}$ 8.5 , yet the alternate conformation occupancy for His200 is different (Mahon et al., 2016). However, our crystals do contain either formate or acetate and the presence of these ligands could be a disrupting factor by altering the 


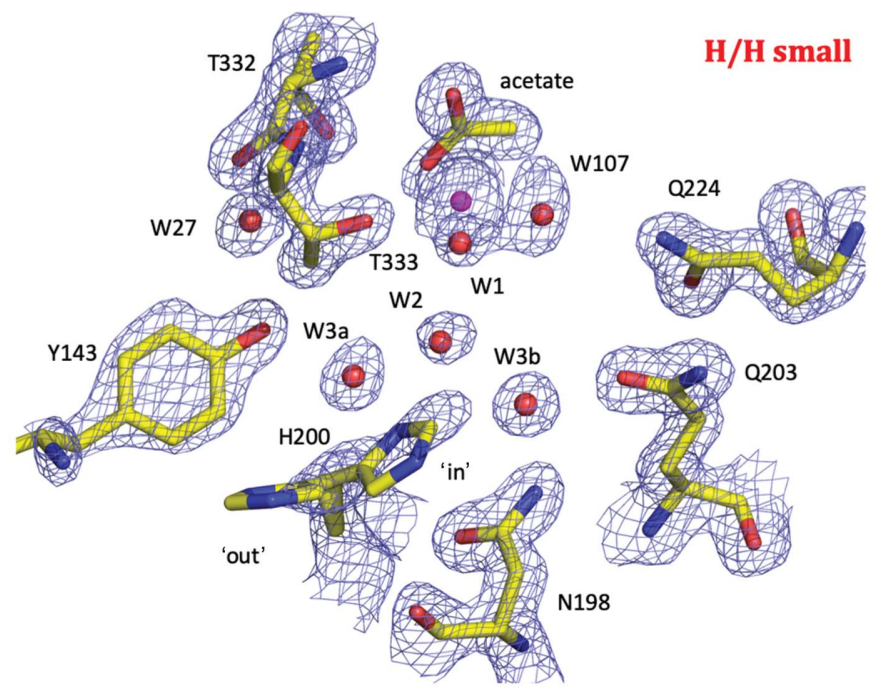

(a)

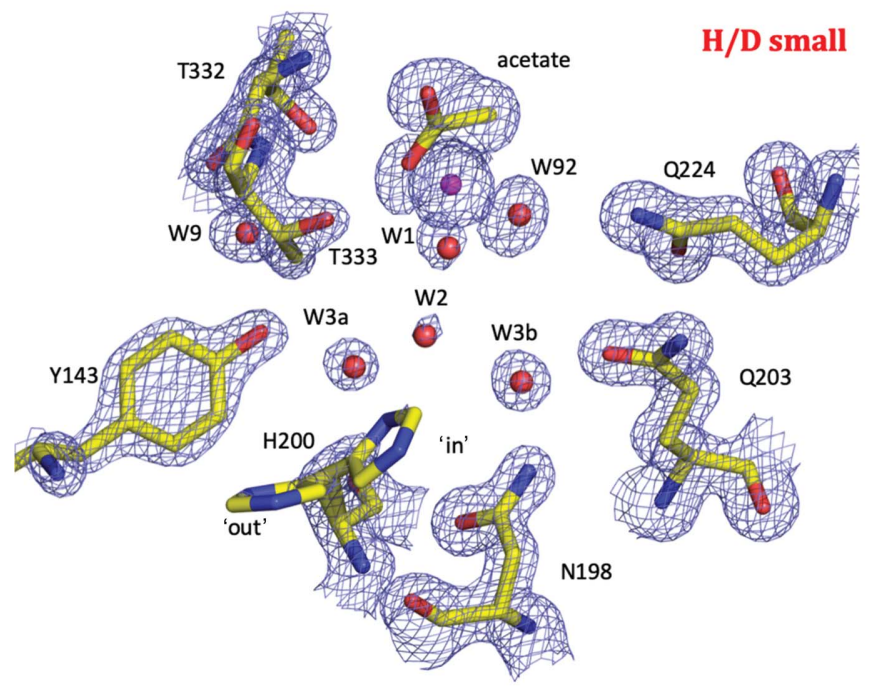

(c)

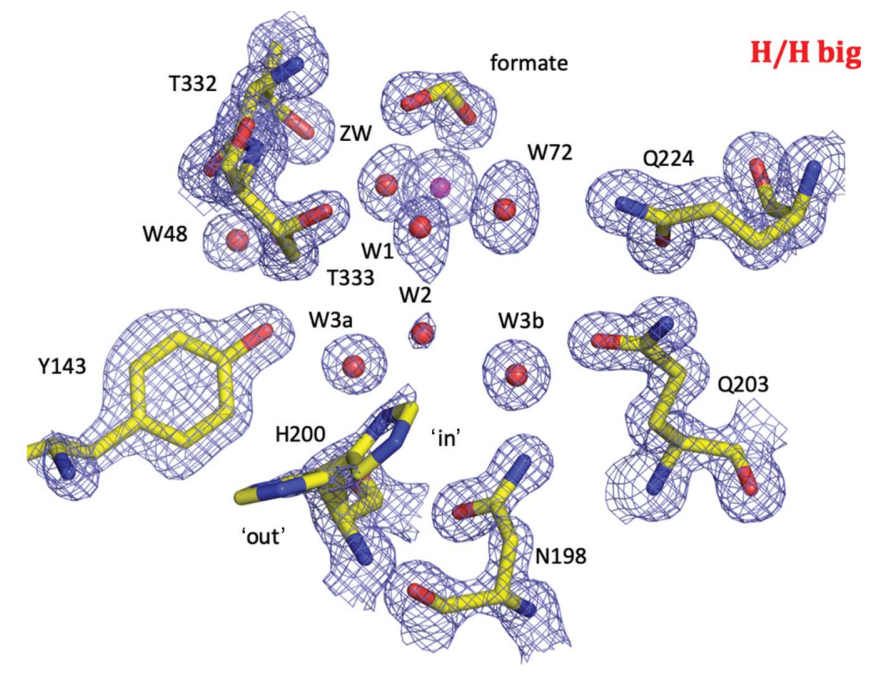

(b)

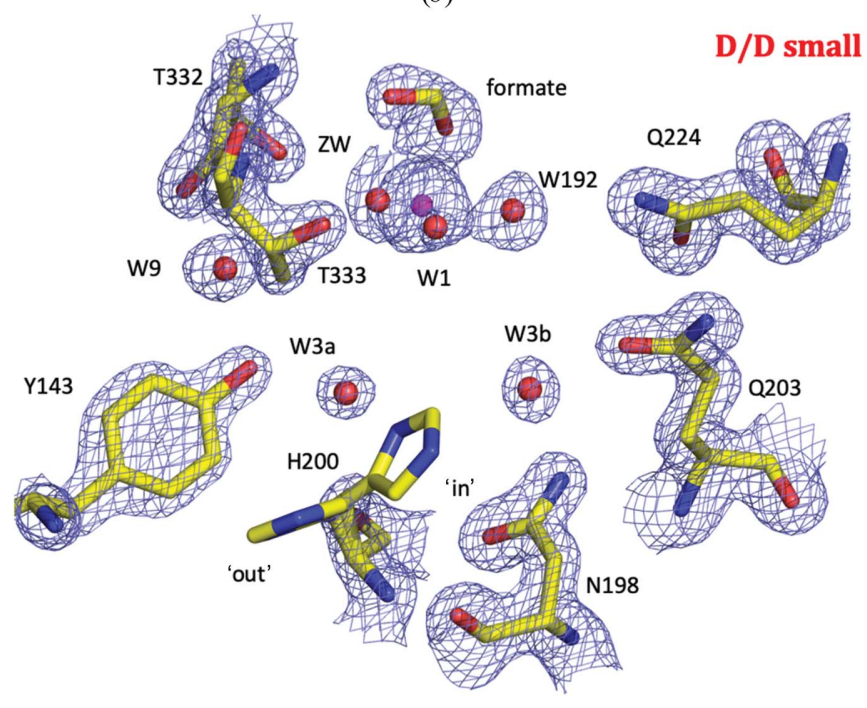

(d)

Figure 5

Active-site comparison of CA IX $\mathrm{Sv}_{\mathrm{Sv}}(a) \mathrm{H} / \mathrm{H}$ in the small $P 2_{1}$ unit cell, $(b) \mathrm{H} / \mathrm{H}$ in the doubled $P 2_{1}$ unit cell, $(c) \mathrm{H} / \mathrm{D}$ exchanged in the small $P 2_{1}$ unit cell and $(d) \mathrm{D} / \mathrm{D}$ CA IX $\mathrm{SV}$ in the small $P 2_{1}$ unit cell. Active-site residues are depicted as yellow sticks; water molecules and $\mathrm{Zn}$ atoms are shown as red and magenta spheres, respectively. $2 F_{\mathrm{o}}-F_{\mathrm{c}}$ electron-density maps are shown in blue mesh and are contoured at $1.50 \sigma$ for residues, $1.25 \sigma$ for solvent and $3.50 \sigma$ for zinc.

electrostatic effect of the zinc charge on the orientation of His64.

Taking the above observations together, for the four structures of the different labelled variants of CA IX $\mathrm{X}_{\mathrm{Sv}}$ we can conclude that deuteration had little to no effect on the overall structure. This is in contrast to other parameters, such as thermal stability and crystallization behaviour, as reported previously (Koruza, Lafumat, Végvári et al., 2018). Furthermore, comparing specific residues that compose the active site shows that the overall architecture is maintained between $\mathrm{H} / \mathrm{H}, \mathrm{H} / \mathrm{D}$ and $\mathrm{D} / \mathrm{D} \mathrm{CA} \mathrm{IX}_{\mathrm{SV}}$.

\section{Conclusions}

Here, we report four crystal structures of different protium/deuterium-labelled versions of $\mathrm{CA} \mathrm{IX}_{\mathrm{SV}}$ in prepara- tion for future neutron crystallographic studies. Despite efforts to reproduce the previously published $P 2_{1} 2_{1} 2_{1}$ crystal form, we instead obtained a different $P 2_{1}$ crystal form from that previously observed for native CA IX and CA IX $\mathrm{Sv}_{\mathrm{Sv}}$. This was an unexpected but fortuitous result, as the unit cell is much smaller than all previously reported for CA IX or CA $\mathrm{IX}_{\mathrm{SV}}$. Overall, the optimized crystallization condition and resulting crystal parameters are more tractable for neutron studies. In addition, the structural comparison of the protiated, partially deuterated and perdeuterated crystal structures reveal that there are insignificant changes owing to deuteration. Hence, the small unit cell $P 2_{1}$ crystals of CA $\mathrm{IX}_{\mathrm{SV}}$ will be used in future neutron crystallographic structural studies for the design of CA IX-specific inhibitors. 


\section{Acknowledgements}

The authors would like to thank Randy Read for critical reading, helpful comments, and for observing the doubling of the small monoclinic unit cell. We would also like to thank Esko Oksanen and Patrick Shaw Stewart for useful discussions. The authors would also like to thank the ESRF (FIPBM30A) and MAX IV laboratory (BioMAX) beamline scientists for expert assistance. We would also like to thank the Lund Protein Production Platform (LP3) staff for providing technical support for experiments and for X-ray data collection. The authors thank the Integrated Infrastructure Initiative No. 262348 European Soft Matter Infrastructure.

\section{Funding information}

We thank Lund University, the Royal Physiographic Society of Lund, Interreg/MAX4ESSFUN, The Crafoord Foundation (award No. 20160528) and BioCARE (a Strategic Research Area at Lund University) for financial support. This project was partly funded by SINE2020.

\section{References}

Adams, P. D., Afonine, P. V., Bunkóczi, G., Chen, V. B., Echols, N., Headd, J. J., Hung, L.-W., Jain, S., Kapral, G. J., Grosse-Kunstleve, R. W., McCoy, A. J., Moriarty, N. W., Oeffner, R. D., Read, R. J., Richardson, D. C., Richardson, J. S., Terwilliger, T. C. \& Zwart, P. H. (2011). Methods, 55, 94-106.

Aggarwal, M., Boone, C. D., Kondeti, B. \& McKenna, R. (2013). J. Enzyme Inhib. Med. Chem. 28, 267-277.

Alterio, V., Hilvo, M., Di Fiore, A., Supuran, C. T., Pan, P., Parkkila, S., Scaloni, A., Pastorek, J., Pastorekova, S., Pedone, C., Scozzafava, A., Monti, S. M. \& De Simone, G. (2009). Proc. Natl Acad. Sci. USA, 106, 16233-16238.

Blakeley, M. P., Hasnain, S. S. \& Antonyuk, S. V. (2015). IUCrJ, 2 , 464-474.

Coleman, J. E. (1967). J. Biol. Chem. 242, 5212-5219.

Di Costanzo, L., Moulin, M., Haertlein, M., Meilleur, F. \& Christianson, D. W. (2007). Arch. Biochem. Biophys. 465, 82-89.

Domsic, J. F. \& McKenna, R. (2010). Biochim. Biophys. Acta, 1804, 326-331.

Duff, A. P., Wilde, K. L., Rekas, A., Lake, V. \& Holden, P. J. (2015). Methods Enzymol. 565, 3-25.

Emsley, P., Lohkamp, B., Scott, W. G. \& Cowtan, K. (2010). Acta Cryst. D66, 486-501.

Fisher, S. J., Blakeley, M. P., Howard, E. I., Petit-Haertlein, I., Haertlein, M., Mitschler, A., Cousido-Siah, A., Salvay, A. G., Popov, A., Muller-Dieckmann, C., Petrova, T. \& Podjarny, A. (2014). Acta Cryst. D70, 3266-3272.

Fisher, S. Z., Aggarwal, M., Kovalevsky, A. Y., Silverman, D. N. \& McKenna, R. (2012). J. Am. Chem. Soc. 134, 14726-14729.

Fisher, S. Z., Maupin, C. M., Budayova-Spano, M., Govindasamy, L., Tu, C., Agbandje-McKenna, M., Silverman, D. N., Voth, G. A. \& McKenna, R. (2007). Biochemistry, 46, 2930-2937.

Fisher, Z., Hernandez Prada, J. A., Tu, C., Duda, D., Yoshioka, C., An, H., Govindasamy, L., Silverman, D. N. \& McKenna, R. (2005). Biochemistry, 44, 1097-1105.
Fisher, Z., Kovalevsky, A. Y., Mustyakimov, M., Silverman, D. N., McKenna, R. \& Langan, P. (2011). Biochemistry, 50, 9421-9423.

Golden, E., Attwood, P. V., Duff, A. P., Meilleur, F. \& Vrielink, A. (2015). Anal. Biochem. 485, 102-108.

Håkansson, K., Carlsson, M., Svensson, L. A. \& Liljas, A. (1992). J. Mol. Biol. 227, 1192-1204.

Hilvo, M., Baranauskiene, L., Salzano, A. M., Scaloni, A., Matulis, D., Innocenti, A., Scozzafava, A., Monti, S. M., Di Fiore, A., De Simone, G., Lindfors, M., Jänis, J., Valjakka, J., Pastoreková, S., Pastorek, J., Kulomaa, M. S., Nordlund, H. R., Supuran, C. T. \& Parkkila, S. (2008). J. Biol. Chem. 283, 27799-27809.

Kabsch, W. (2010). Acta Cryst. D66, 133-144.

Kazokaitè, J., Niemans, R., Dudutienè, V., Becker, H. M., Leitāns, J., Zubrienè, A., Baranauskienè, L., Gondi, G., Zeidler, R., Matulienè, J., Tars, K., Yaromina, A., Lambin, P., Dubois, L. J. \& Matulis, D. (2018). Oncotarget, 9, 26800-26816.

Kim, C. U., Song, H., Avvaru, B. S., Gruner, S. M., Park, S. \& McKenna, R. (2016). Proc. Natl Acad. Sci. USA, 113, 5257-5262.

Koruza, K., Lafumat, B., Nyblom, M., Knecht, W. \& Fisher, S. Z. (2018). Crystals, 8, 434-445.

Koruza, K., Lafumat, B., Végvári, Á., Knecht, W. \& Fisher, S. Z. (2018). Arch. Biochem. Biophys. 645, 26-33.

Kovalevsky, A., Aggarwal, M., Velazquez, H., Cuneo, M. J., Blakeley, M. P., Weiss, K. L., Smith, J. C., Fisher, S. Z. \& McKenna, R. (2018). Structure, 26, 383-390.

Krissinel, E. \& Henrick, K. (2007). J. Mol. Biol. 372, 774-797.

Kuijk, S. J. A. van, Yaromina, A., Houben, R., Niemans, R., Lambin, P. \& Dubois, L. J. (2016). Front. Oncol. 6, 69.

Langan, P. \& Chen, J. C.-H. (2013). Phys. Chem. Chem. Phys. 15, 13705-13712.

Langella, E., Buonanno, M., Vullo, D., Dathan, N., Leone, M., Supuran, C. T., De Simone, G. \& Monti, S. M. (2018). Cell. Mol. Life Sci. 75, 3283-3296.

Li, Y., Wang, H., Tu, C., Shiverick, K. T., Silverman, D. N. \& Frost, S. C. (2011). Biochim. Biophys. Acta, 1813, 159-167.

Liu, X., Hanson, B. L., Langan, P. \& Viola, R. E. (2007). Acta Cryst. D63, 1000-1008.

Mahon, B. P., Bhatt, A., Socorro, L., Driscoll, J. M., Okoh, C., Lomelino, C. L., Mboge, M. Y., Kurian, J. J., Tu, C., AgbandjeMcKenna, M., Frost, S. C. \& McKenna, R. (2016). Biochemistry, 55, 4642-4653.

Mahon, B. P., Pinard, M. A. \& McKenna, R. (2015). Molecules, 20, 2323-2348.

McCoy, A. J., Grosse-Kunstleve, R. W., Adams, P. D., Winn, M. D., Storoni, L. C. \& Read, R. J. (2007). J. Appl. Cryst. 40, 658-674.

Meilleur, F., Coates, L., Cuneo, M. J., Kovalevsky, A. \& Myles, D. A. A. (2018). Crystals, $\mathbf{8}, 388$.

Nair, S. K. \& Christianson, D. W. (1991). J. Am. Chem. Soc. 113, 9455 9458.

O'Dell, W. B., Bodenheimer, A. M. \& Meilleur, F. (2016). Arch. Biochem. Biophys. 602, 48-60.

Pastorek, J. \& Pastorekova, S. (2015). Semin. Cancer Biol. 31, 52-64.

Pinard, M. A., Mahon, B. P. \& McKenna, R. (2015). Biomed Res. Int. 2015, 453543.

Roth, M., Carpentier, P., Kaïkati, O., Joly, J., Charrault, P., Pirocchi, M., Kahn, R., Fanchon, E., Jacquamet, L., Borel, F., Bertoni, A., Israel-Gouy, P. \& Ferrer, J.-L. (2002). Acta Cryst. D58, 805-814.

Silverman, D. N. \& McKenna, R. (2007). Acc. Chem. Res. 40, 669-675. Tanaka, I. (2019). JPS Conf. Proc. 25, 011015.

Vonrhein, C., Flensburg, C., Keller, P., Sharff, A., Smart, O., Paciorek, W., Womack, T. \& Bricogne, G. (2011). Acta Cryst. D67, 293-302. 